\title{
Comparison of Additives in Anode: The Case of Graphene, MXene, CNTs Integration with Silicon Inside Carbon Nanofibers
}

\author{
Min Jiang ${ }^{1} \cdot$ Miaomiao Jiang ${ }^{1} \cdot$ Hong Gao $^{2}$. Junliang Chen ${ }^{1} \cdot$ Wuming Liu ${ }^{1} \cdot$ Yuanyuan Ma ${ }^{1} \cdot$ Wei Luo ${ }^{1}$. \\ Jianping Yang ${ }^{1}$
}

Received: 27 July 2020 / Revised: 18 August 2020 / Accepted: 2 September 2020 / Published online: 28 October 2020

(c) The Chinese Society for Metals (CSM) and Springer-Verlag GmbH Germany, part of Springer Nature 2020

\begin{abstract}
Recently, graphene oxide (GO), MXene, carbon nanotubes (CNTs) have been used for compounding with other materials as anodes and cathodes to achieve excellent electrochemical properties for metal-ion batteries. However, few researches have focused on the differences between the three additives. Herein, silicon, as a typical anode, is selected to integrate with MXene, GO and CNTs in carbon nanofibers (CNFs) and form Si/MXene@CNFs, Si/GO@CNFs and Si/CNTs@CNFs, respectively. Together with the results, it can be realized that these CNFs with a significant improved performance compared with pure $\mathrm{Si} @ \mathrm{CNFs}$ show superiority in different aspects of electrochemical properties. Additionally, the reasons for the superiority are also discussed in this work. The addition of MXene can improve the cycle stability of the electrodes, thereby obtaining a high capacity retention rate, CNTs are favorable for the enhancement of rate performance, and the electrodes reversible capacity can be increased due to the addition of GO. Consequently, the studies on three additives may contribute to the rational design of silicon-based and other anode materials.
\end{abstract}

Keywords Silicon alloy $\cdot$ Graphene oxide $\cdot$ MXene $\cdot$ Carbon nanotubes $\cdot$ Lithium-ion battery

\section{Introduction}

With the dramatic increase in demand for various applications, such as electric vehicles and various energy storage systems, higher requirements have paced on energy density and cycle life of batteries [1,2]. Recently, alloy materials

Available online at https://link.springer.com/journal/40195.

Electronic supplementary material The online version of this article (https://doi.org/10.1007/s40195-020-01153-6) contains supplementary material, which is available to authorized users.

Yuanyuan Ma

yyma@dhu.edu.cn

$\triangle$ Jianping Yang

jianpingyang@dhu.edu.cn

1 State Key Laboratory for Modification of Chemical Fibers and Polymer Materials, College of Materials Science and Engineering, Donghua University, Shanghai 201620, China

2 State Key Laboratory of Advanced Special Steel, Shanghai Key Laboratory of Advanced Ferrometallurgy, Shanghai University, Shanghai 200444, China have attracted much attention and proved to be promising anodes on account of their high energy density, thus widely used in metal-ion batteries (such as $\mathrm{Li}, \mathrm{Na}, \mathrm{K}, \mathrm{Mg}$ ) [3-10]. Among all alloy materials used for lithium ion batteries (LIBs) anodes, silicon-based alloys are considered as the promising candidate anode materials due to its superior theoretical capacity, suitable lithium (Li) intercalation potential $\left(\sim 0.2 \mathrm{~V}\right.$ vs. $\left.\mathrm{Li} / \mathrm{Li}^{+}\right)$and high abundance $[11,12]$. The high capacity of silicon results from the formation of the Li-Si alloy $\left(\mathrm{Li}_{22} \mathrm{Si}_{5}: 4200 \mathrm{~mA} \mathrm{~h} \mathrm{~g}{ }^{-1} ; \mathrm{Li}_{15} \mathrm{Si}_{4}: 3579 \mathrm{~mA} \mathrm{~h}\right.$ $\mathrm{g}^{-1}$ ) [13]. However, the practical application of silicon-based alloy anodes is still seriously hindered by several thorny issues, including large volume change $(\sim 300 \%)$, unstable solid-electrolyte interphase and low intrinsic electronic and ionic conductivity, which may lead to rapid deterioration and capacity loss of electrodes [14, 15].

Plentiful efforts have been proposed to figure out these problems [15-18]. As one of the effective methods that can improve the electrochemical performance of silicon-based anodes, nanometerization can effectively relieve volume expansion, shorten the ion diffusion path, improve the electrochemical activity of materials and reduce the polarization of electrochemical reactions $[19,20]$. Compounding nano-silicon 
with highly conductive materials (such as graphene, MXene, carbon nanotubes and carbon nanofibers) is another effective and promising measure to improve the properties of siliconbased electrodes [21, 22]. In particular, graphene, MXene and carbon nanotubes (CNTs) as high conductive additives have been used to compound with silicon due to their unique properties of high specific surface area, outstanding mechanical properties and excellent conductivity [23-27]. Therefore, the composites formed by combining graphene/MXene/ CNTs with silicon show excellent $\mathrm{Li}$-storage capability with high capacity, fast ion diffusion and good structural stability [28-32]. For instance, Zhou and co-workers fabricated a SiNP@G nanocomposite using positively-charged silicon nanoparticles and negatively-charged graphene as raw materials by a self-assembly method, which manifested stable cycling performance and excellent rate capability [33]. Tian and coworkers used silicon nanoparticles and MXene to prepare a self-supporting silicon/MXene composite by a simple suction filtration method, which exhibited superior electrochemical performance with a steady cycling capacity of $1672 \mathrm{~mA} \mathrm{~h} \mathrm{~g}^{-1}$ at $1000 \mathrm{~mA} \mathrm{~g}^{-1}$ after 200 cycles [34]. Ma and co-works developed a CNTs@silicon composite with coaxial hollow nanocables structure by chemical vapor deposition (CVD) [35]. However, most of current researches focus on single material and there are few comparative studies on the advantages of MXene, graphene and CNTs.

Herein, we compare the effects of graphene oxide (GO), MXene (the typical representation: $\mathrm{Ti}_{3} \mathrm{C}_{2} \mathrm{~T}_{x}$ ) and CNTs on silicon in the composites with the assistance of carbon nanofibers (CNFs), where silicon nanoparticles are embedded in combined conductive network of MXene/GO/CNTs additives and carbon matrix to obtain Si/MXene@CNFs, Si/GO@CNFs and Si/CNTs@CNFs. To ensure fair comparison, MXene, GO, CNTs and silicon nanoparticles are uniformly mixed at the same mass ratio to prepare the spinning solution. After preoxidation and carbonization, various structures and properties of these CNFs are characterized to distinguish the effects of these additives on silicon. When used as anodes for LIBs, Si/ CNTs@CNFs exhibit a superior rate performance with a high reversible capacity of $820.1 \mathrm{~mA} \mathrm{~h} \mathrm{~g}^{-1}$ at $5 \mathrm{~A} \mathrm{~g}^{-1}$. Additionally, the reversible capacity can return to $1419.2 \mathrm{~mA} \mathrm{~h} \mathrm{~g}^{-1}$ with a high restore rate of $93 \%$ when the setting is restored to 0.1 $\mathrm{A}^{-1}$. The Si/MXene@CNFs shows excellent cycle stability with a capacity retention of $48 \%$ at $1 \mathrm{~A} \mathrm{~g}^{-1}$ after 200 cycles. As for Si/GO@CNFs, a high reversible capacity of $623.2 \mathrm{~mA} \mathrm{~h}$ $\mathrm{g}^{-1}$ at $1 \mathrm{~A} \mathrm{~g}^{-1}$ after 200 cycles can be obtained.

\section{Experimental}

\subsection{Preparation of $\mathrm{Ti}_{3} \mathrm{C}_{2} \mathrm{~T}_{x}$ Nanosheets, GO Nanosheets and CNTs}

Typically, $\mathrm{Ti}_{3} \mathrm{C}_{2} \mathrm{~T}_{x}$ nanosheets were prepared by etching of $\mathrm{Ti}_{3} \mathrm{AlC}_{2}$ with $\mathrm{LiF}$ and $\mathrm{HCl}$ [36]. The GO nanosheets were fabricated from natural graphite flake using a modified Hummers method [37]. The commercial CNTs were treated with the combination of concentrated $\mathrm{H}_{2} \mathrm{SO}_{4}$ and $\mathrm{HNO}_{3}$ at a ratio of 3:1 for $3 \mathrm{~h}$ to obtain active CNTs [38].

\subsection{Preparation of Si/MXene@CNFs, Si/GO@CNFs and Si/CNTs@CNFs}

Briefly, $0.10 \mathrm{~g}$ of $\mathrm{Ti}_{3} \mathrm{C}_{2} \mathrm{~T}_{x}$ (GO/CNTs) and $0.20 \mathrm{~g}$ of commercial silicon were dissolved into $4 \mathrm{~mL}$ of $N, N$-dimethylformamide (DMF) to get uniform solution. Then, $0.42 \mathrm{~g}$ of polyacrylonitrile (PAN) was added into the solution and vigorously stirred at $50{ }^{\circ} \mathrm{C}$ for $12 \mathrm{~h}$. The resulting solution, which placed in the 5 -mL syringe with an 18 -gauge blunt tip needle, was subjected to electrospinning at a flow rate of $0.7-1 \mathrm{~mL} \mathrm{~min}^{-1}$ with a voltage of $12-14 \mathrm{kV}$. The nanofibers were received by a drum rotating at a distance

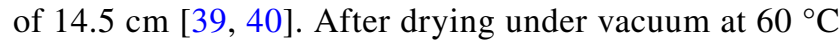
to remove the solvent, the nanofibers were placed in a tube furnace for pre-oxidation $\left(5^{\circ} \mathrm{C} \mathrm{min}{ }^{-1}\right.$, stabilized at $260{ }^{\circ} \mathrm{C}$ for $2 \mathrm{~h}$, air) and carbonized $\left(2{ }^{\circ} \mathrm{C} \mathrm{min}-1\right.$, maintained at $600{ }^{\circ} \mathrm{C}$ for $2 \mathrm{~h}, \mathrm{Ar}$ ) to obtain Si/MXene@CNFs, Si/GO@ CNFs and Si/CNTs@CNFs. In addition, the synthesis process of Si@CNFs is the same as the above process except for the addition of the additives.

\subsection{Materials Characterization}

The surface morphology and internal structure morphology of these nanofibers were characterized by a field emission scanning electron microscope (FESEM, TESCAN MAIA3), a field emission transmission electron microscope (FETEM, FEI Talos F200S) and a high-resolution transmission electron microscope (HRTEM). The crystallinity and crystal structure of these CNFs were characterized by X-ray diffraction (XRD, Rigaku D/Max-2550 PC). The degree of graphitization of these CNFs was mainly determined by a Raman spectrometer (Dilor LabRam1B). Thermogravimetric analysis (TGA, TG209F1 Libra) was performed in air atmosphere with the heating rate of $10 \mathrm{~K} \mathrm{~min}^{-1}$ to test the relative content of each component in the CNFs. The composition and valence state of the 
elements in these CNFs were characterized by X-ray photoelectron spectroscopy (XPS, Escalab 250Xi).

\subsection{Measurements of Electrochemical Performances}

According to the ratio of active material: carboxymethyl cellulose (CMC): and super $P=7: 1.5: 1.5$, the uniform slurry was coated on the copper foil and dried in a vacuum oven at $80{ }^{\circ} \mathrm{C}$ for $12 \mathrm{~h}$ to obtain the work electrodes with loading mass of $0.8-1.1 \mathrm{mg} \mathrm{cm}^{-2}$. After transferring the active material-coated electrodes into the glove box, the CR2032 coin-type LIBs were assembled in the glove box filled with argon. The electrolyte was 1.0 M LiPF6 in 3:4:3 (weight ratio) of ethylene carbonate/dimethyl carbonate/ diethylene carbonate, with $5 \mathrm{wt} \%$ fluoroethylene carbonate additives. Galvanostatic charge-discharge (GCD) tests were performed at the Neware battery test system with a voltage range of 0.01 to $2.0 \mathrm{~V}$ ( $\left(\mathrm{vs} \mathrm{Li} / \mathrm{Li}^{+}\right.$). The cyclic voltammetry (CV) was measured at the Biologic VMP-3 electrochemical workstation with a scan rate of $0.1 \mathrm{mV} \mathrm{s}^{-1}$. Electrochemical impedance spectroscopy (EIS) was performed at the same type electrochemical workstation with the frequency of $0.01 \mathrm{~Hz}-100 \mathrm{kHz}$.

\section{Results and Discussion}

Figure 1 is the synthesis process of three CNFs. First, the silicon nanoparticles were separately dispersed in DMF with GO, MXene and CNTs at the same mass ratio, respectively. PAN powder was subsequently added in the homogeneously mixed mixture to obtain uniform spinning solution. Then, PAN nanofibers were produced by electrospinning and the collected nanofibers were stabilized in air. Finally, the nanofibers were pyrolyzed at $600{ }^{\circ} \mathrm{C}$ for $2 \mathrm{~h}$ under an argon atmosphere, where PAN was decomposed into carbon. Fig. S1a-d shows the SEM images of $\mathrm{Ti}_{3} \mathrm{AlC}_{2}$, $\mathrm{Ti}_{3} \mathrm{C}_{2} \mathrm{~T}_{x}$, GO and CNTs, respectively. Compared with layered $\mathrm{Ti}_{3} \mathrm{AlC}_{2}, \mathrm{Ti}_{3} \mathrm{C}_{2} \mathrm{~T}_{x}$ exhibits a distinct monolayer structure. Meanwhile, the XRD patterns in Fig. S2a also show the disappearance of (104) and left shift of (002) diffraction peak, representing the successful exfoliation of monolayer $\mathrm{Ti}_{3} \mathrm{C}_{2} \mathrm{~T}_{x}$. The SEM image and XRD pattern in Fig. S2b of GO nanosheets also show a monolayer structure and obvious (002) diffraction peak like $\mathrm{Ti}_{3} \mathrm{C}_{2} \mathrm{~T}_{x}$. In the SEM images of Si/MXene@CNFs,Si/GO@CNFs and Si/CNTs@CNFs shown in Fig. 2d-f, silicon nanoparticles and additives can be seen on the surface of CNFs compared with the PAN nanofibers shown in Fig. 2a-c. These are also verified by the TEM and HRTEM images in Fig. 3a-f, in which lattice fringes spacing of $\sim 0.32 \mathrm{~nm}$ belonging to the (111) plane of silicon can be found in three CNFs. What is more, the (100) plane of CNTs with a lattice fringe spacing of $0.362 \mathrm{~nm}$ can also be observed in the HRTEM image of Si/CNTs@CNFs. Furthermore, the diameter of these CNFs can be observed from TEM images, where the diameter of Si/MXene@CNFs ( 200-230 nm) is significantly smaller than that of Si/GO@CNFs ( 740-800 nm) and Si/CNTs@ CNFs ( 420-450 nm). High-angle annular dark-field scanning transmission electron microscopy (HAADF-STEM) and the corresponding energy-dispersive $\mathrm{X}$-ray spectrometry (EDS) element mapping images of Si/MXene@CNFs, Si/ GO@CNFs and Si/CNTs@CNFs are displayed in Figs.3g, S3 and S4, respectively. Obviously, the C, N and Si elements are homogeneously distributed in these CNFs, indicating that the silicon nanoparticles not only exist on the surface of these CNFs, but also are abundant in the interior of CNFs.

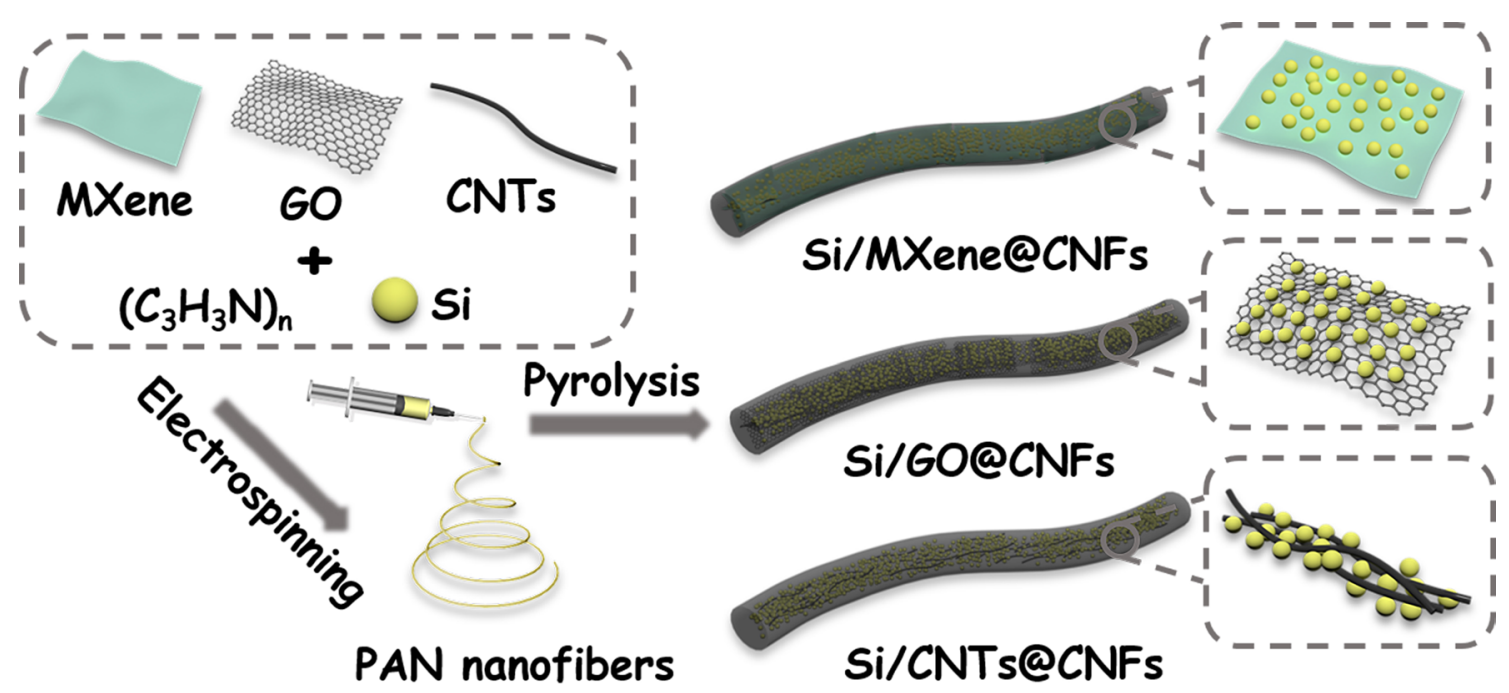

Fig. 1 Schematic illustration of the preparation procedure of Si/MXene@CNFs, Si/GO@CNFs and Si/CNTs@CNFs 

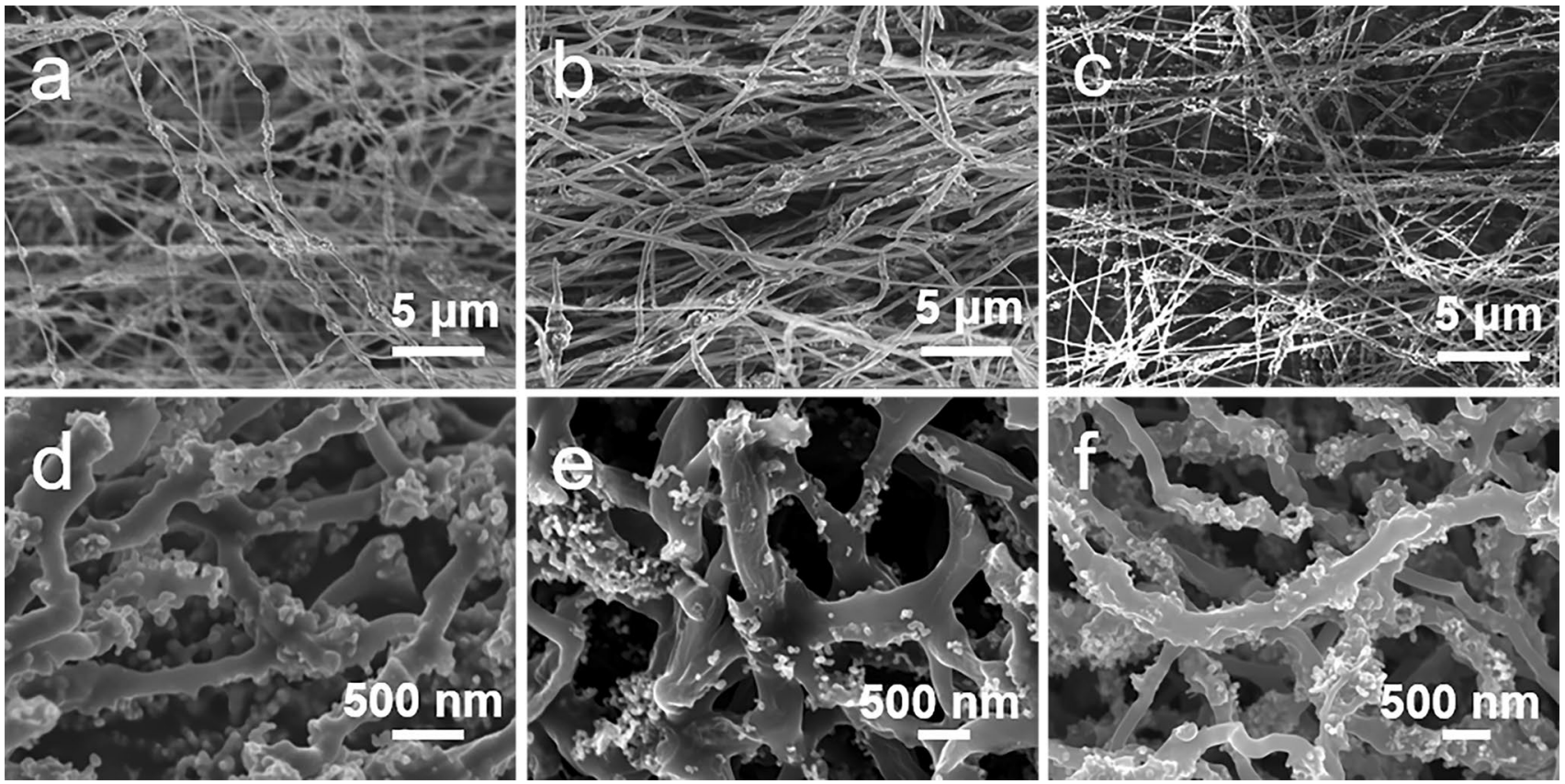

Fig. 2 SEM images of Si/MXene@PAN nanofibers a, Si/GO@PAN nanofibers b, Si/CNTs@PAN nanofibers c, Si/MXene@CNFs d, Si/GO@ CNFs e, Si/CNTs@CNFs f

In addition, there are evenly distributed Ti elements in the Si/MXene@CNFs, which represents the widely existence of $\mathrm{Ti}_{3} \mathrm{C}_{2}$ in the CNFs. These additives are compounded with silicon nanoparticles and embedded in the carbon matrix which can be demonstrated by the apparent images, but the information of chemical structure is required for further verification.

The XRD patterns of Si/MXene@CNFs,Si/GO@CNFs and Si/CNTs@CNFs are exhibited in Fig. 4a, which both show obvious (111), (220), (311) and (331) peaks of crystalline silicon (JCPDS No. 27-1402). Additionally, a broad peak belonging to $\mathrm{SiO}_{x}$ at $\sim 24^{\circ}$ can be seen in the XRD pattern of Si/MXene@CNFs. Owing to the well dispersion and low contents of GO and $\mathrm{Ti}_{3} \mathrm{C}_{2} \mathrm{~T}_{x}$, there is no evident (002) peak in these XRD patterns [41, 42]. Raman spectra of these CNFs in Fig. 4b both show sharp silicon peaks and broad G-band $\left(\sim 1358 \mathrm{~cm}^{-1}\right)$ and D-band peaks $\left(\sim 1580 \mathrm{~cm}^{-1}\right)$ [43]. The small shift of silicon peak in these CNFs can be attributed to the carbon matrix in which silicon nanoparticles are confined $[44,45]$. The $I_{\mathrm{G}} / I_{\mathrm{D}}$ of Si/MXene@CNFs, Si/GO@ CNFs and Si/CNTs@CNFs, which can be calculated by the intensity ratio of $\mathrm{G}$ peak and $\mathrm{D}$ peak, are about $1.12,1,22$ and 1.24 , respectively, indicating the low graphitic degree of these CNFs. Furthermore, the maximum $I_{\mathrm{G}} / I_{\mathrm{D}}$ value of Si/CNTs@CNFs indicates that more defects are introduced into the CNFs, which is favorable for the diffusion of $\mathrm{Li}$ ions $[46,47]$. From the TGA curves shown in Fig. 4c, it can be observed that the weight loss interval of carbon is between 450 and $650{ }^{\circ} \mathrm{C}$, and the mass increase at high temperature may be ascribed to the oxidation of silicon. The silicon content of Si/CNTs@CNFs can be easily calculated to be $51.4 \%$ due to the simple carbon material composition [48]. However, the presence of $\mathrm{Ti}_{3} \mathrm{C}_{2}$ oxide and GO residues in the other two CNFs results in a slight increase in content (Si/MXene@CNFs: 60.5\%,Si/GO@CNFs: 55.5\%). According to the TGA curve of Si@CNFs shown in Fig. S5, the theoretical silicon content of these CNFs can be deduced to be $\sim 47.2 \%$, which is roughly in line with the previous calculation. The XPS measurement was also performed to test the as-prepared CNFs. As shown in Fig. 4d, it is easy to find that there is no Ti $2 p$ peak in the XPS full spectra except for Si/MXene@CNFs. In the C $1 s$ spectra of these CNFs (Fig. 4e), two peaks located at 284.8 and $\sim 286.3 \mathrm{eV}$ can be assigned to $\mathrm{C}-\mathrm{C}$ bond and $\mathrm{Si}-\mathrm{C}$ bond, and the highest intensity C-C can be seen in the Si/CNT@CNFs. Additionally, Si/MXene@CNFs displays an extra peak at $289.8 \mathrm{eV} \mathrm{cor-}$ responding to $\mathrm{C}-\mathrm{Ti}$ bond, which demonstrates the existence of $\mathrm{Ti}_{3} \mathrm{C}_{2}$ [49]. In the $\mathrm{Si} 2 p$ spectra of the Si/GO@CNFs and Si/CNTs@CNFs (Fig. 4f), Si-Si bond centered at $99.9 \mathrm{eV}$ can be clearly seen, and $\mathrm{Si}-\mathrm{O}$ bond appears at $\sim 103.7 \mathrm{eV}$ due to the slight oxidation of silicon [50]. As for Si/MXene@ $\mathrm{CNFs}$, in addition to the $\mathrm{Si}-\mathrm{Si}$ bond, there are peaks at 102.4 and $103.5 \mathrm{eV}$ that belong to $\mathrm{Si} / \mathrm{SiO}_{x}(0<x<2)$ [46]. Compared with other additives, the formation of $\mathrm{SiO}_{x}$ may be attributed to the low hydroxyl group content and poor thermostability of $\mathrm{Ti}_{3} \mathrm{C}_{2}$, which combines with silicon during the pre-oxidation process, causing partial oxidation $[51,52]$. In summary, these characterizations indicate the composition 


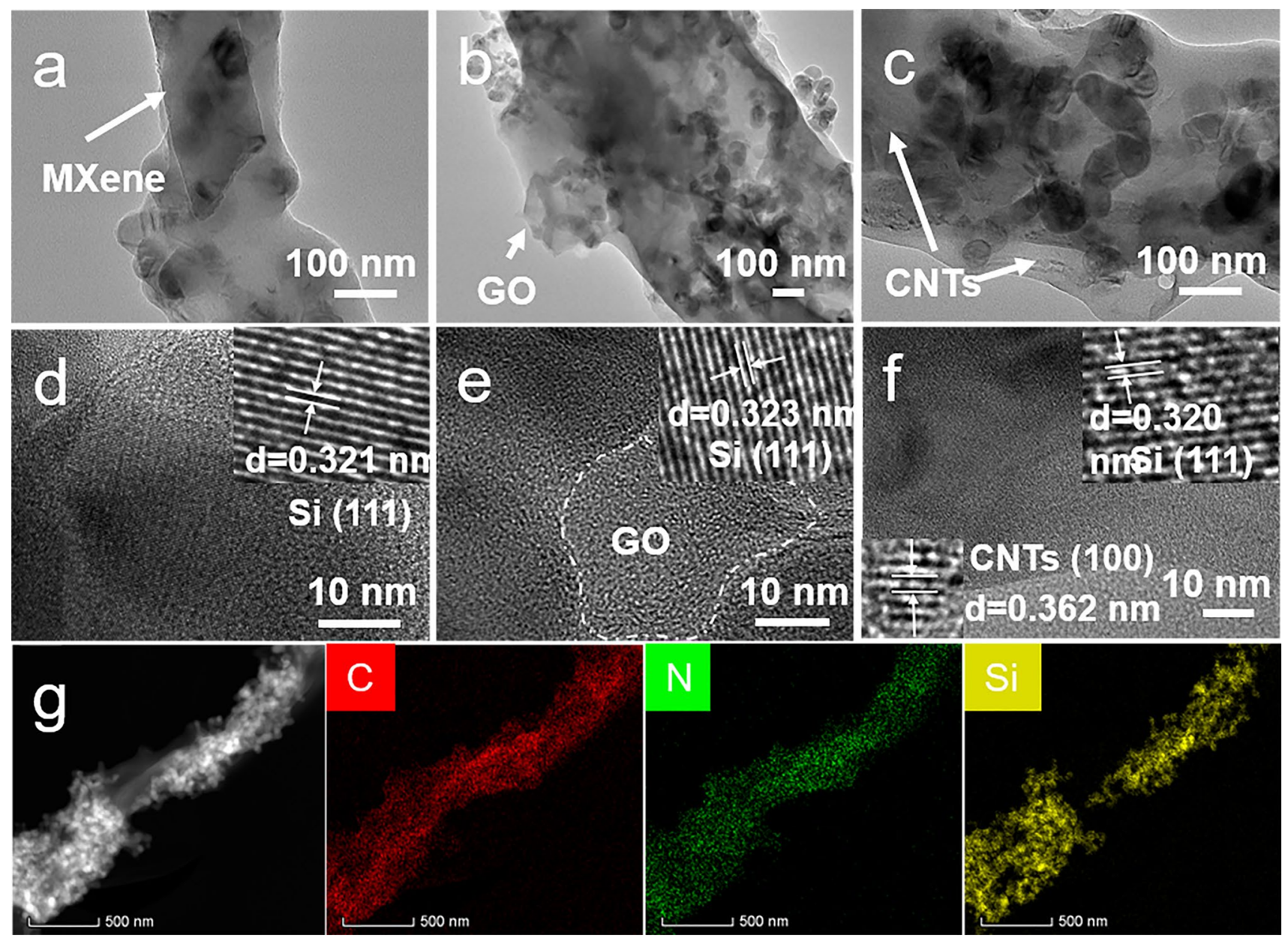

Fig. 3 TEM and HRTEM images of Si/MXene@CNFs a, d, Si/GO@CNFs b, e, Si/CNTs@CNFs c, f; HAADF-STEM image and the corresponding EDS element mapping of Si/CNTs@CNFs $\mathbf{g}$

of these CNFs, including silicon, carbon and additives (MXene, GO and CNTs).

To compare the electrochemical performance of these CNFs, CV and GCD measurements were tested. Figure 5a-c shows the CV curves of Si/MXene@CNFs, Si/ GO@CNFs and Si/CNTs@CNFs electrodes, respectively. In the first cathode sweep of these CNFs anodes, a broad peak appears at $\sim 0.54 \mathrm{~V}$ and disappears in the next scan, which can be ascribed to the formation of solid-electrolyte interphase (SEI) film that causes the irreversible loss in the first cycle. A reduction peak at $\sim 0.14 \mathrm{~V}$ may correspond to the alloying process between silicon and $\mathrm{Li}$. In the anodic sweep, there are two strong oxidation peaks at $\sim 0.32 \mathrm{~V}$ and $\sim 0.54 \mathrm{~V}$, which may belong to the formation process from $\mathrm{Si}-\mathrm{Li}$ alloy to amorphous silicon [53]. The average initial specific discharge/charge capacities of Si/MXene@ CNFs, Si/GO@CNFs and Si/CNTs@CNFs anodes are 1348.3/1775.6, 1647.4/2111.2 and 1490.1/1958.5 $\mathrm{mA} \mathrm{h} \mathrm{g}^{-1}$ with the average initial coulombic efficiency (ICE) of $75.9 \%$, $78.0 \%$ and $76.1 \%$, respectively (Fig. 5d-f). Obviously, the
Si/GO@CNFs anode shows an advantage on the initial specific capacities, which may be caused by the high capacity of GO with abundant oxygen-containing group [54]. The low capacity of Si/MXene @CNFs can be attributed to the existence of $\mathrm{SiO}_{x}$ with lower capacity than silicon. Moreover, the ICE of three CNFs is close, which means that adding these additives may have little effect on ICE. As shown in Fig. $5 \mathrm{~g}-\mathrm{i}$, the GCD profiles of these CNFs at the initial three cycles all exhibit a broad discharge voltage platform of about $0.1 \mathrm{~V}$, which belongs to the lithiation process of silicon [55]. It can be seen from the above results that there is no apparent difference in ICE, which means that the rate property and long cycle performance should be tested.

To compare the rate capability of these CNFs, the electrodes are cycled for five times in sequence from 0.1 , $0.2,0.5,1,2$ to $5 \mathrm{~A} \mathrm{~g}^{-1}$, and finally return to the first setting current density. As described in Fig. 6a, the reversible capacities of Si/MXene@CNFs, Si/GO@CNFs, Si/ CNTs@CNFs anodes can maintain at 1502.4/1614.8/1526.7, 1426.8/1398.0/1376.6, 1220.5/1177.4/1256.9, 

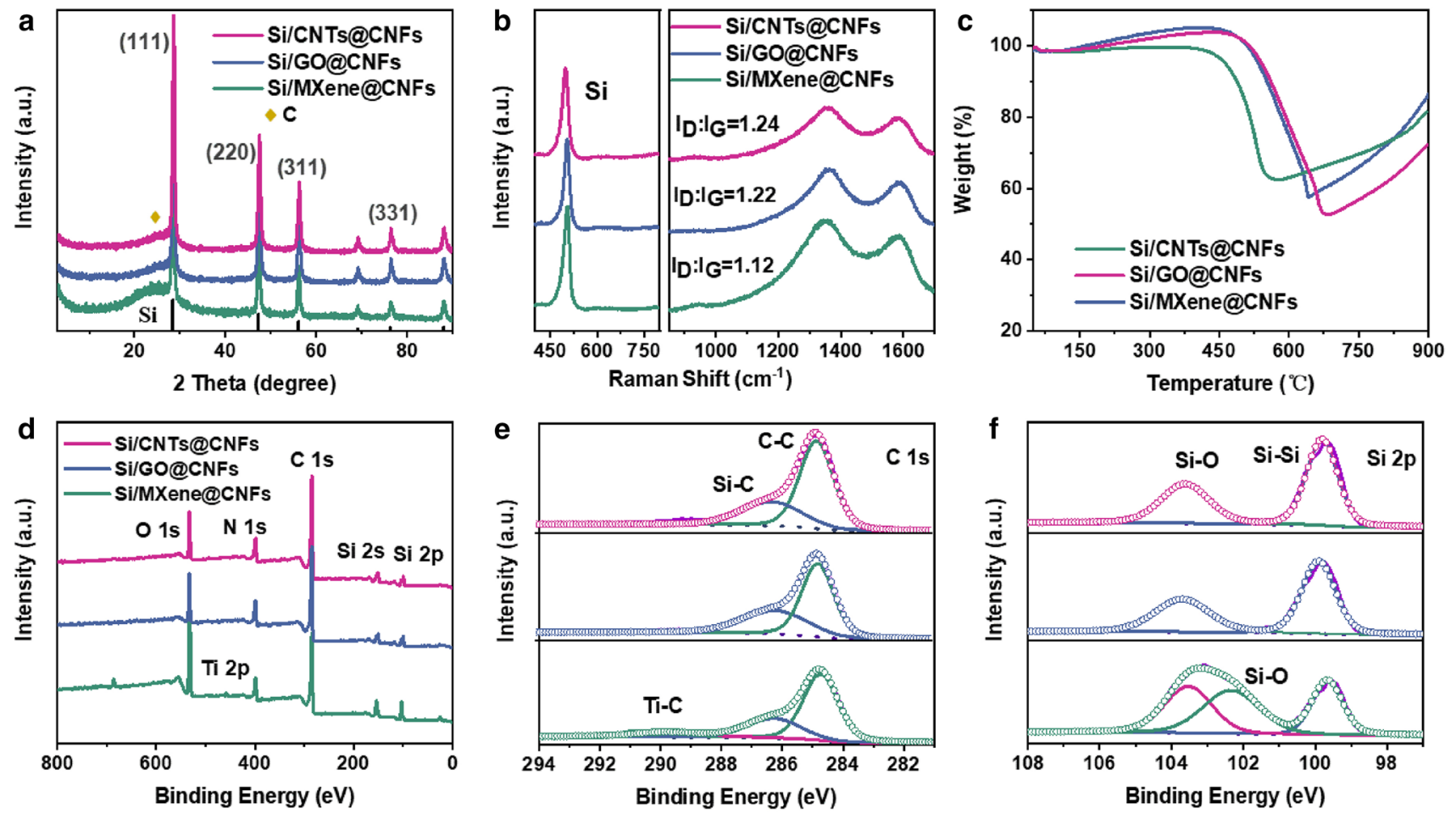

Fig. 4 XRD patterns a, Raman patterns b, TGA curves $\mathbf{c}$, XPS spectra d, C $1 s$ XPS spectra e, Si $2 p$ XPS spectra f of Si/MXene@CNFs, Si/ GO@CNFs and Si/CNTs@CNFs

931.2/944.2/1018.8, 728.2/693.7/913.3, 648.1/630.3/820.1 and $1342.9 / 1375.7 / 1419.2 \mathrm{~mA} \mathrm{~h} \mathrm{~g}^{-1}$, respectively. Although the specific capacity of Si/CNTs@CNFs anode shows disadvantage at low current density, it is higher than Si/MXene@ CNFs and Si/CNTs@CNFs anodes at high current density. Even tested at $5 \mathrm{~A} \mathrm{~g}^{-1}$, the capacity retention capability of Si/CNTs@CNFs can still reach 42.6\%, and a high capacity recovery rate of $93.0 \%$ can be obtained when the current density returns from 5 to $0.1 \mathrm{~A} \mathrm{~g}^{-1}$ (Fig. 6c). In comparison, the rate performance of $\mathrm{Si} / \mathrm{GO} @ \mathrm{CNF}$ is disadvantage, which may be related to the higher Li-ion barrier compared with other additives [30]. In the cycle performance of these CNFs at a current density of $1 \mathrm{~A} \mathrm{~g}^{-1}$ shown in Fig. 6b, the reversible capacities of Si/MXene@CNFs, Si/GO@CNFs and Si/CNTs@CNFs anodes remain at 440.3, 623.2 and $589.5 \mathrm{~mA} \mathrm{~h} \mathrm{~g}^{-1}$ after 200 cycles, respectively. Apparently, the Si/GO@CNFs anode delivers a higher reversible capacity than other CNFs. Nevertheless, the reversible capacity value after cycling is not the only criterion for the electrode cycling stability because the initial capacities of these anodes are different. Therefore, the capacity retention rate requires to be calculated to distinguish the CNFs anode with the best capacity stability, which is shown in Fig. 6d. It is worth noting that regardless of the cycling numbers, the reversible capacity and capacity retention rate of these CNFs all are higher than those of Si@CNFs (Fig. S6a), demonstrating the improvement in these additives for silicon-based anode. At the current density of $1 \mathrm{~A} \mathrm{~g}^{-1}$, the capacity retention rates of Si/MXene@CNFs,Si/GO@CNFs,Si/CNTs@CNFs anodes are almost the same after 30 cycles and decrease with the increase in cycle numbers. Obviously, it can be perceived that the capacity retention rate of Si/MXene@ CNFs anode is higher than those of other anodes showing similar capacity retention rate. The capacity retention rate of Si/MXene@CNFs can maintain at 48\% even after 200 cycles, indicating the best capacity stability compared to other anodes. In addition, these CNFs anodes are also tested at $0.5 \mathrm{~A} \mathrm{~g}^{-1}$ shown in Fig. S6b, c, and the Si/MXene@CNFs anode also delivers low capacity and high capacity retention rate. A suitable reason for the excellent cycling stability may be the existence of $\mathrm{SiO} x$, which can act as a buffering layer to alleviate the volume change, thereby maintaining the electrode integrity $[44,56,57]$. Based on the above results, the rate performance, cycle stability and capacity (include initial capacity and cycled capacity) of these CNFs anodes are divided into three levels: high $(\mathrm{H})$, medium $(\mathrm{M})$ and low (L) for comparison. As shown in Fig. 6e, the rate properties, cycle stabilities and capacities of Si/MXene@CNFs, Si/ GO@CNFs and Si/CNTs@CNFs anodes are M/H/L, L/M/H and $\mathrm{H} / \mathrm{M} / \mathrm{M}$, respectively. According to the above results, the CNFs anodes display different electrochemical performance superiorities due to different additives. If only capacity is 

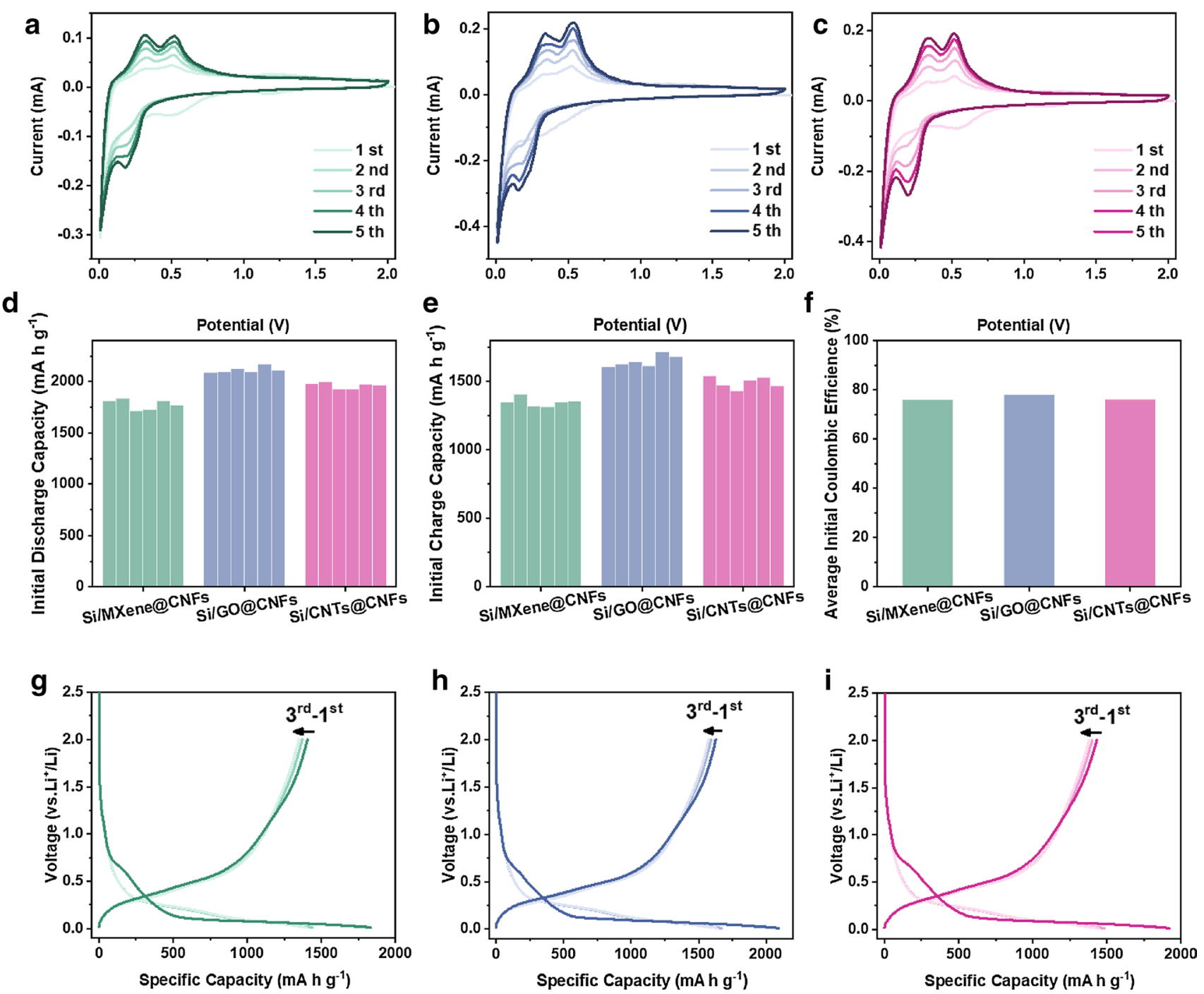

Fig. 5 CV curves of Si/MXene@CNFs a, Si/GO@CNFs b, Si/CNTs@CNFs c electrodes for the first five cycles at a scan rate of $0.1 \mathrm{mV} \mathrm{s}^{-1}$ in the voltage range of $0.01-2 \mathrm{~V}\left(\mathrm{vs} \mathrm{Li} / \mathrm{Li}^{+}\right)$; the initial discharge capacity $\mathbf{d}$, initial charge capacity $\mathbf{e}$, average ICE $\mathbf{f}$ of Si/MXene@CNFs, Si/GO@ CNFs and Si/CNTs@CNFs; the GCD in the first three cycles of Si/MXene@CNFs g, Si/GO@CNFs h, Si/CNTs@CNFs i anodes at 0.1 A g ${ }^{-1}$

considered, Si/GO@CNFs may be the best choice. However, the Si/MXene@CNFs may be the best candidate when taking rate property and cycle stability into consideration.

Although the electrochemical performances of these CNFs anodes have been compared, the reasons for the superiority are still unknown. Therefore, the $\mathrm{CV}$ measurement is performed at various scan rates ranging from 0.1 to $2.0 \mathrm{mV} \mathrm{s}^{-1}$ to get the $\mathrm{Li}$-storage mechanisms of these CNFs. The Li-storage mechanism can be evaluated by the following equation: $i=a v^{b}$, where $i$ is the peak current and $v$ is the corresponding scan rate [58]. Remarkably, the $b$ value, obtained from the slope of the linear relationship between $\log (i)$ and $\log (v)$, can determine the Li-storage mechanism. A value of 0.5 represents the diffusion-controlled battery behavior, while 1.0 represents the surface capacitance-controlled pseudocapacitance behavior.
Furthermore, the Li-storage mechanism depends on how close $b$ is to 0.5 and 1 when $b$ is an intermediate value. The former represents the battery mechanism mainly controlled by diffusion-controlled process, and the latter indicates the pseudocapacitance behavior based on surface capacitance process [52, 59]. According to CV curves shown in Fig. 7a-c, the calculated $b$ values of Si/MXene@CNFs, Si/GO@CNFs and Si/CNTs@CNFs anodes are 0.87, 0.85, 0.88, respectively (Fig. 7d-f). These CNFs display similar Li-storage mechanism, which is the pseudocapacitance mechanism mainly controlled by surface capacitance process. Moreover, the contribution of pseudocapacitance to the total capacity can be obtained by the formula: $i=k_{1} v+k_{2} v^{\frac{1}{2}}$, where $k_{1} v$ and $k_{2} v^{\frac{1}{2}}$ indicate the surface capacitance-controlled contribution and diffusioncontrolled contribution, respectively [30,60]. Consequently, 

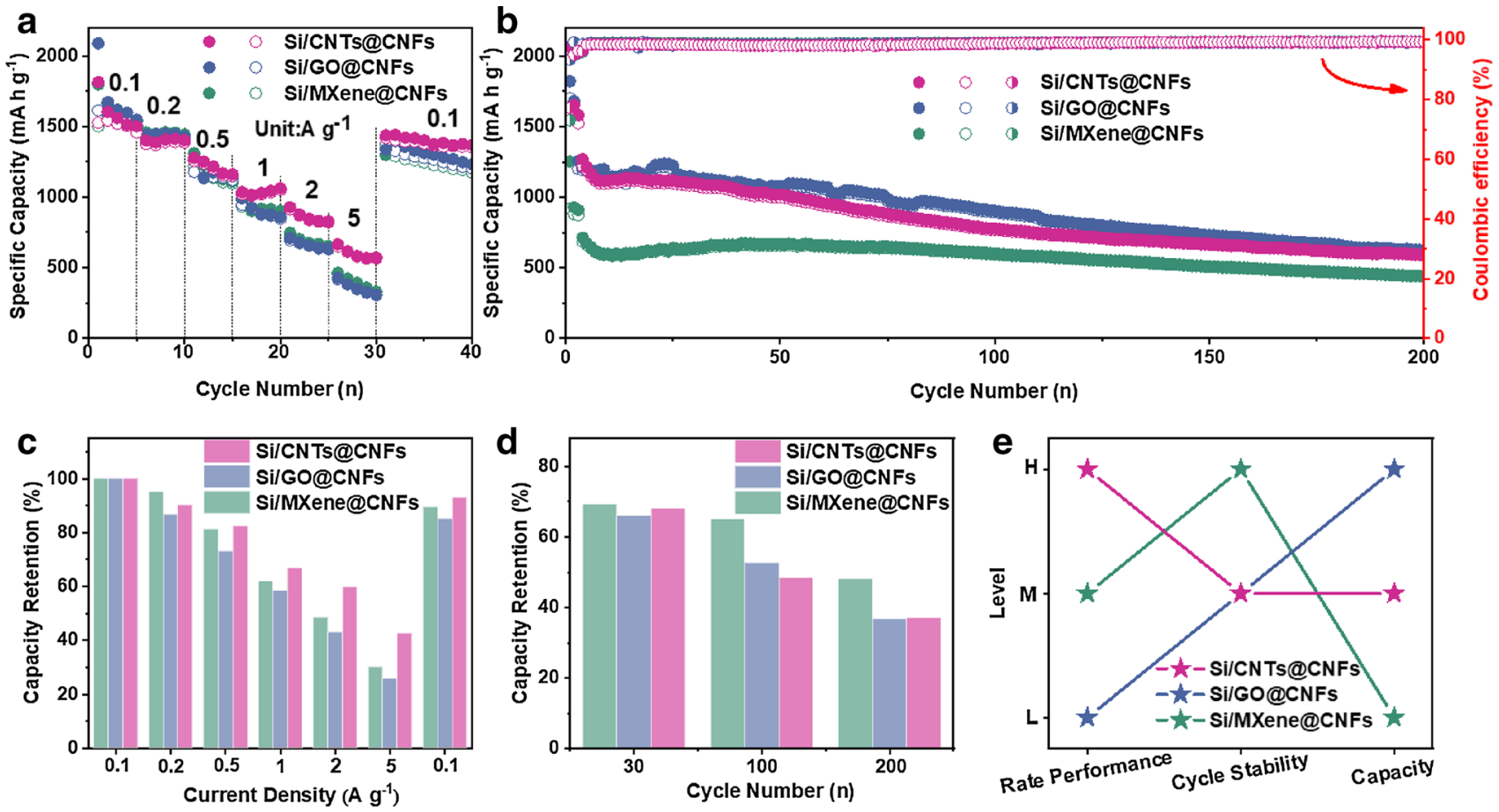

Fig. 6 Rate performance a, capacity retention rate $\mathbf{c}$ at different current densities of Si/MXene@CNFs, Si/GO@CNFs and Si/CNTs@CNFs anodes; cycle performance of the three CNFs at current density of $1 \mathrm{~A} \mathrm{~g}^{-1} \mathbf{b}$, the corresponding capacity retention rate after different cycles $\mathbf{d}$; comparison of the electrochemical performance of three CNFs anodes $\mathbf{e}$
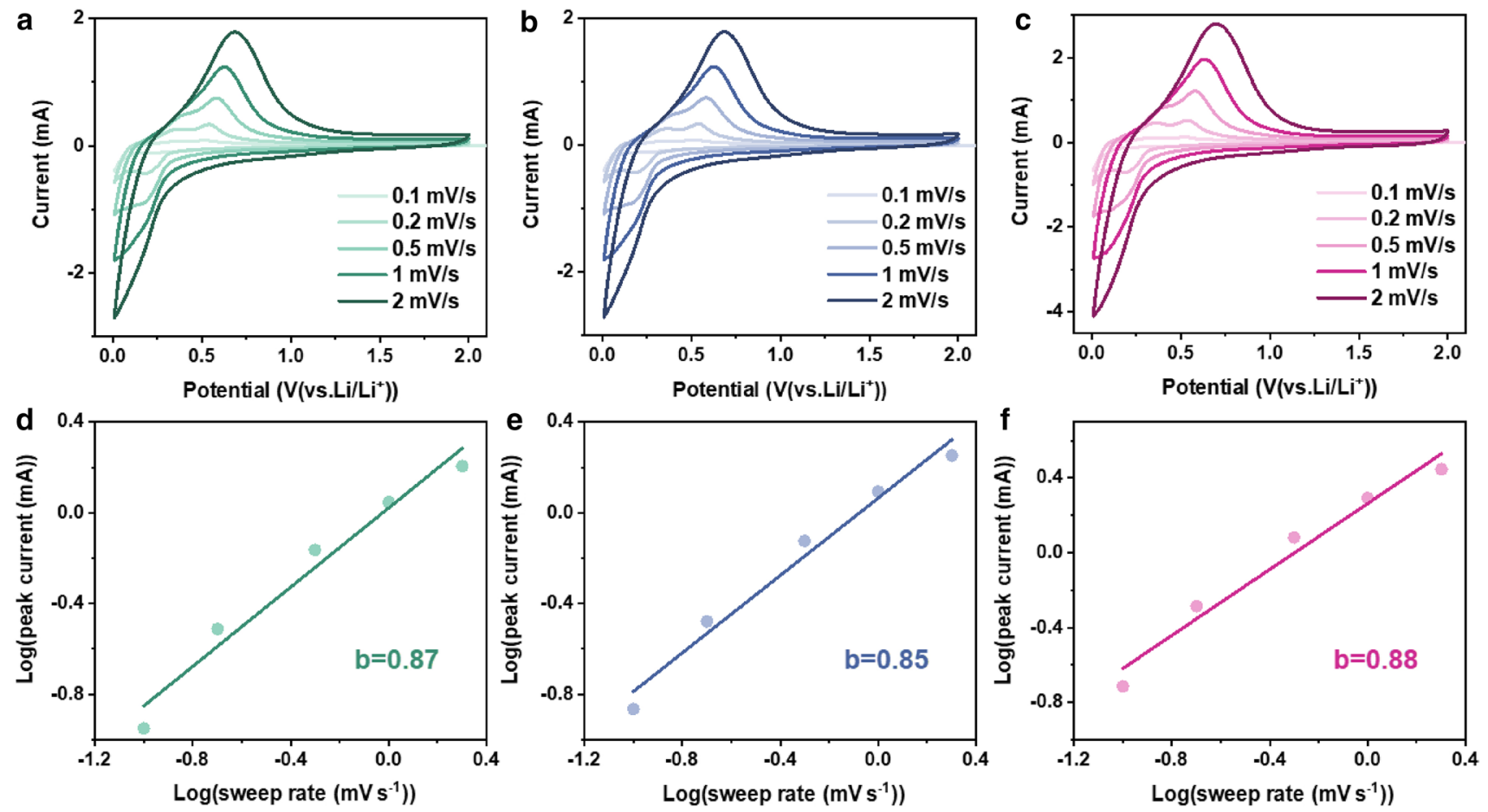

Fig. 7 CV curves of Si/MXene@CNFs a, Si/GO@CNFs b, Si/CNTs@CNFs c anodes at various scan rates ranging from 0.1 to $2.0 \mathrm{mV} \mathrm{s} \mathrm{s}^{-1}$ and the corresponding linear relationships between the peak current and the scan rate of Si/MXene@CNFs d, Si/GO@CNFs e, Si/CNTs@CNFs $\mathbf{f}$ anodes 

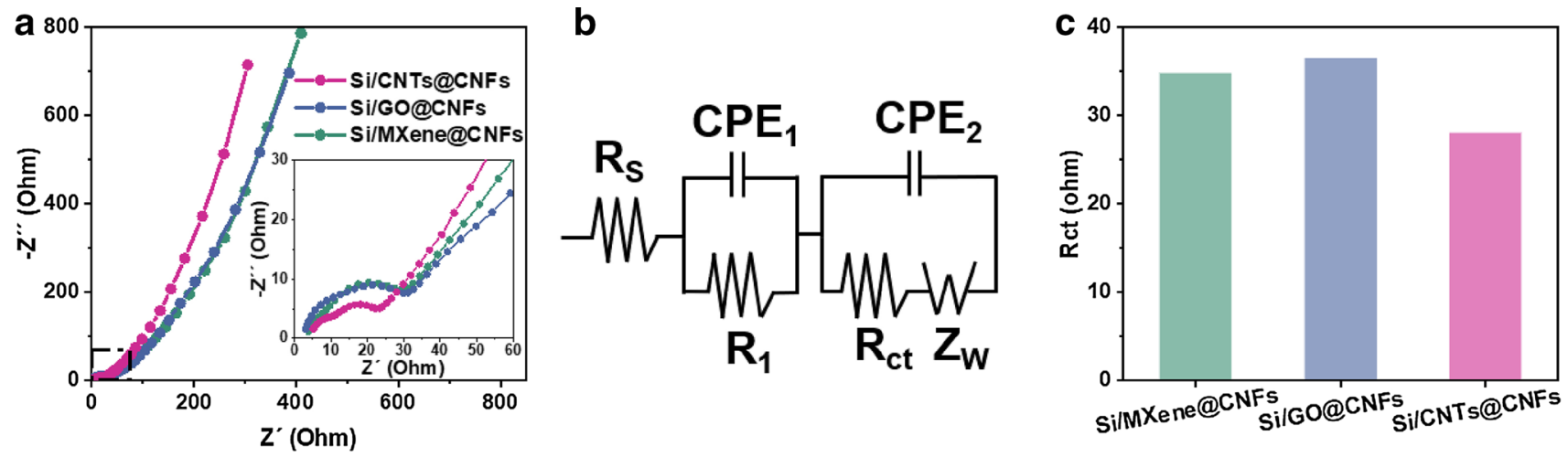

Fig. 8 EIS a, simulated equivalent circuit of the Si/MXene@CNFs, Si/GO@CNFs and Si/CNTs@CNFs b, $R_{\mathrm{ct}}$ of three CNFs electrodes after 15 cycles c

the $b$ value of Si/CNTs@CNFs anode is higher than those of others, indicating that it has more pseudocapacitance contribution. The result can be verified by Fig. S7a-c, the surface capacitance-controlled contribution of all electrodes increases with the increase in scan rate, and Si/CNTs@CNFs can reach $88.7 \%$ when tested at a scan rate of $1 \mathrm{mV} \mathrm{s}^{-1}$. The pseudocapacitance is favorable for enhancing the kinetics of $\mathrm{Li}$ ions, thereby improving the rate and $\mathrm{Li}$-storage performance properties. The high pseudocapacitance contribution can be one of the reasons for the high rate performance of Si/CNTs@CNFs.

The above conclusion also can be supported by the Nyquist plots. All curves are composed of a semicircle at high frequency and a slanted line in the low-frequency region. The straight line in the low-frequency region corresponds to Li-diffusion, and the high-frequency semicircle is related to the charge transfer resistance. In EIS shown in Fig. $8 \mathrm{a}$, the order of the high-frequency semicircles diameters of the three electrodes is: Si/CNTs@CNFs $<\mathrm{Si} /$ MXene@CNFs<Si/GO@CNFs. According to the above result, the simulated equivalent circuit can be obtained (Fig. 8b). Three electrodes all conform to the same equivalent circuit, in which $\mathrm{CPE}_{1}$ and $R_{1}$ are the capacitance and resistance of SEI film, $R_{\mathrm{s}}, \mathrm{CPE}_{2}, R_{\mathrm{ct}}$ and $Z_{\mathrm{W}}$ are the ionic resistance of the electrolyte, double-layer capacitance, charge transfer resistance and Warburg impedance, respectively. The $R_{\mathrm{ct}}$ values of these Si/MXene@CNFs, Si/GO@ CNFs and Si/CNTs@CNFs electrodes are 34.8, 36.5 and 28.1, respectively (Fig. 8c). The low charge transfer resistance can promote the transmission of $\mathrm{Li}$ ions, thus improving the rate property, which is consistent with previous tests.

\section{Conclusion}

In this work, different additives including MXene, GO and CNTs, and silicon were distributed in carbon matrix by electrospinning method to explore the influence of these additives on silicon-based anodes. Compared with Si@CNFs anode, the electrochemical performance has been significantly enhanced, but the specific aspects of the improvement are different according to different additives. The excellent cycle stability, medium rate property can be obtained in $\mathrm{Si}$ / MXene@CNFs anode due to medium pseudocapacitance contribution and the existence of $\mathrm{SiO}_{x}$, which also leads to low capacity. Owing to the low charge transfer resistance, the Si/CNTs@CNFs shows excellent rate performance. In addition, the Si/GO@CNFs anode delivers high reversible capacity even at a current density of $1 \mathrm{~A} \mathrm{~g} \mathrm{~g}^{-1}$. As a result, the well-designed CNFs not only reveals the different effects of the three additives on silicon, but also shows excellent Li-storage performance, which also provides new insight into the design of other silicon-based anodes with a required property.

Acknowledgements This work was financially supported by the National Natural Science Foundation of China (No. 51702046), the Open Project of State Key Laboratory of Advanced Special Steel, Shanghai Key Laboratory of Advanced Ferrometallurgy, Shanghai University (SKLASS 2019-2021) and the Science and Technology Commission of Shanghai Municipality (No. 19DZ2270200), the Program for Professor of Special Appointment (Eastern Scholar) at Shanghai Institutions of Higher Learning, State Key Laboratory for Modification of Chemical Fibers and Polymer Materials, Donghua University.

\section{References}

[1] Z. Wei, B. Ding, H. Dou, J. Gascon, X.J. Kong, Y. Xiong, B. Cai, R. Zhang, Y. Zhou, M. Long, J. Miao, Y. Dou, D. Yuan, J. Ma. Chin. Chem. Lett. 30, 2110 (2019)

[2] H. Liu, X. Liu, W. Li, X. Guo, Y. Wang, G. Wang, D. Zhao, Adv. Energy Mater. 7, 1700283 (2017)

[3] J. Su, C. Zhang, X. Chen, S. Liu, T. Huang, A. Yu, J. Power Sources 381, 66 (2018)

[4] W. Ma, K. Yin, H. Gao, J. Niu, Z. Peng, Z. Zhang, Nano Energy 54, 349 (2018)

[5] L. Xue, H. Gao, W. Zhou, S. Xin, K. Park, Y. Li, J.B. Goodenough, Adv. Mater. 28, 9608 (2016) 
[6] Y.H. Tan, W.T. Yao, T. Zhang, T. Ma, L.L. Lu, F. Zhou, H.B. Yao, S.H. Yu, ACS Nano 12, 5856 (2018)

[7] Y.S. Hong, C.Z. Zhao, Y. Xiao, R. Xu, J.J. Xu, J.Q. Huang, Q. Zhang, X. Yu, H. Li, Batter. Supercaps 2, 638 (2019)

[8] X. Zhang, A. Chen, M. Jiao, Z. Xie, Z. Zhou, Batter. Supercaps 2, 498 (2019)

[9] X. Yang, Y.Y. Wang, B.H. Hou, H.J. Liang, X.X. Zhao, H. Fan, G. Wang, X.L. Wu, Acta Metall. Sin.-Engl. Lett. (2020). https:// doi.org/10.1007/s40195-020-01001-7

[10] S. Liu, J. Mao, Q. Zhang, Z. Wang, W.K. Pang, L. Zhang, A. Du, V. Sencadas, W. Zhang, Z. Guo, Angew. Chem. Int. Ed. 59, 3638 (2020)

[11] N. Liu, H. Wu, M.T. McDowell, Y. Yao, C. Wang, Y. Cui, Nano Lett. 12, 3315 (2012)

[12] S.H. Ng, J. Wang, D. Wexler, K. Konstantinov, Z.P. Guo, H.K. Liu, Angew. Chem. Int. Ed. 45, 6896 (2006)

[13] P. Li, J.Y. Hwang, Y.K. Sun, ACS Nano 13, 2624 (2019)

[14] W. Luo, X. Chen, Y. Xia, M. Chen, L. Wang, Q. Wang, W. Li, J. Yang, Adv. Energy Mater. 7, 1701083 (2017)

[15] J. Yang, G. Zhu, F. Zhang, X. Li, W. Luo, L. Li, H. Zhang, L. Wang, Y. Wang, W. Jiang, H.K. Liu, S.X. Dou, Angew. Chem. Int. Ed. 58, 6669 (2019)

[16] J. Wu, Y. Cao, H. Zhao, J. Mao, Z. Guo, Carbon Energy 1, 57 (2019)

[17] C. Zhang, R. Yu, T. Zhou, Z. Chen, H. Liu, Z. Guo, Carbon 72, 169 (2014)

[18] X. Chen, X.Q. Zhang, H.R. Li, Q. Zhang, Batter. Supercaps 2, 128 (2019)

[19] J. Liu, N.P. Wickramaratne, S.Z. Qiao, M. Jaroniec, Nat. Mater. 14, 763 (2015)

[20] H. Tian, J. Liang, J. Liu, Adv. Mater. 31, 1903886 (2019)

[21] B. Xu, S. Qi, M. Jin, X. Cai, L. Lai, Z. Sun, X. Han, Z. Lin, H. Shao, P. Peng, Z. Xiang, J.E. ten Elshof, R. Tan, C. Liu, Z. Zhang, X. Duan, J. Ma, Chin. Chem. Lett. 30, 2053 (2019)

[22] X. Ma, G. Hou, Q. Ai, L. Zhang, P. Si, J. Feng, L. Ci, Sci. Rep. 7, 9642 (2017)

[23] R. Rojaee, R. Shahbazian-Yassar, ACS Nano 14, 2628 (2020)

[24] T. Li, L. Yao, Q. Liu, J. Gu, R. Luo, J. Li, X. Yan, W. Wang, P. Liu, B. Chen, W. Zhang, W. Abbas, R. Naz, D. Zhang, Angew. Chem. Int. Ed. 57, 6115 (2018)

[25] J. Nan, X. Guo, J. Xiao, X. Li, W. Chen, W. Wu, H. Liu, Y. Wang, M. Wu, G. Wang, Small 16, 1902085 (2020)

[26] F. Guo, P. Chen, T. Kang, Y.L. Wang, C.H. Liu, Y.B. Shen, W. Lu, L.W. Chen, Acta Phys.-Chim. Sin. 35, 1365 (2019)

[27] H.F. An, L. Jiang, F. Li, P. Wu, X.S. Zhu, S.H. Wei, Y.M. Zhou, Acta Phys.-Chim. Sin. 36, 1905034 (2020)

[28] Z. Liu, S.P. Lau, F. Yan, Chem. Soc. Rev. 44, 5638 (2015)

[29] Y. Zhang, Z. Mu, J. Lai, Y. Chao, Y. Yang, P. Zhou, Y. Li, W. Yang, Z. Xia, S. Guo, ACS Nano 13, 2167 (2019)

[30] X. Hui, R. Zhao, P. Zhang, C. Li, C. Wang, L, Yin. Adv. Energy Mater. 9, 1901065 (2019)

[31] J. Pang, R.G. Mendes, A. Bachmatiuk, L. Zhao, H.Q. Ta, T. Gemming, H. Liu, Z. Liu, M.H. Rummeli, Chem. Soc. Rev. 48, 72 (2019)

[32] X. Li, L. Zhi, Chem. Soc. Rev. 47, 3189 (2018)

[33] X. Zhou, Y.X. Yin, L.J. Wan, Y.G. Guo, Adv. Energy Mater. 2, $1086(2012)$
[34] Y. Tian, Y. An, J. Feng, A.C.S. Appl, Mater. Interfaces 11, 10004 (2019)

[35] T. Ma, H. Xu, X. Yu, H. Li, W. Zhang, X. Cheng, W. Zhu, X. Qiu, ACS Nano 13, 2274 (2019)

[36] F. Shahzad, M. Alhabeb, C.B. Hatter, B. Anasori, S.M. Hong, C.M. Koo, Y. Gogotsi, Science 353, 1137 (2016)

[37] W.S. Hummers, R.E. Offeman, J. Am. Chem. Soc. 80, 1339 (1958)

[38] I.D. Rosca, F. Watari, M. Uo, T. Akasaka, Carbon 43, 3124 (2005)

[39] X. Zhou, Y.G. Guo, J. Mater. Chem. A 1, 9019 (2013)

[40] A.S. Levitt, M. Alhabeb, C.B. Hatter, A. Sarycheva, G. Dion, Y. Gogotsi, J. Mater. Chem. A 7, 269 (2019)

[41] X. Zhou, Y.X. Yin, L.J. Wan, Y.G. Guo, Chem. Commun. 48 $2198(2012)$

[42] X. Zhu, J. Shen, X. Chen, Y. Li, W. Peng, G. Zhang, F. Zhang, X. Fan, Chem. Eng. J. 378, 122212 (2019)

[43] F. Zhang, G. Zhu, K. Wang, X. Qian, Y. Zhao, W. Luo, J. Yang, J. Mater. Chem. A 7, 17426 (2019)

[44] Y.S. Hu, R. Demir-Cakan, M.M. Titirici, J.O. Müller, R. Schlögl, M. Antonietti, J. Maier, Angew. Chem. Int. Ed. 47, 1645 (2008)

[45] M.S. Park, E. Park, J. Lee, G. Jeong, K.J. Kim, J.H. Kim, Y.J. Kim, H. Kim, A.C.S. Appl, Mater. Interfaces 6, 9608 (2014)

[46] Y. Chen, Y. Hu, Z. Shen, R. Chen, X. He, X. Zhang, Y. Zhang, K. Wu, Electrochim. Acta 210, 53 (2016)

[47] L. Fei, B.P. Williams, S.H. Yoo, J. Kim, G. Shoorideh, Y.L. Joo, A.C.S. Appl, Mater. Interfaces 8, 5243 (2016)

[48] M.S. Wang, W.L. Song, L.Z. Fan, ChemElectroChem 2, 1699 (2015)

[49] M. Jiang, F. Zhang, G. Zhu, Y. Ma, W. Luo, T. Zhou, J. Yang, A.C.S. Appl, Mater. Interfaces 12, 24796 (2020)

[50] J. Guo, G. Zhao, T. Xie, D. Dong, C. Ma, L. Su, L. Gong, X. Lou, X. Guo, J. Wang, Y. Zhu, A.C.S. Appl, Mater. Interfaces 12, $19023(2020)$

[51] M. Seredych, C.E. Shuck, D. Pinto, M. Alhabeb, E. Precetti, G. Deysher, B. Anasori, N. Kurra, Y. Gogotsi, Chem. Mater. 31, 3324 (2019)

[52] D. Zhao, R. Zhao, S. Dong, X. Miao, Z. Zhang, C. Wang, L. Yin, Energy Environ. Sci. 12, 2422 (2019)

[53] W. Ye, F. Pei, X. Lan, Y. Cheng, X. Fang, Q. Zhang, N. Zheng, D.L. Peng, M.S. Wang, Adv. Energy Mater. 10, 1902956 (2020)

[54] D.R. Dreyer, S. Park, C.W. Bielawski, R.S. Ruoff, Chem. Soc. Rev. 39, 228 (2010)

[55] H. Wang, J. Fu, C. Wang, J. Wang, A. Yang, C. Li, Q. Sun, Y. Cui, H. Li, Energy Environ. Sci. 13, 848 (2020)

[56] G. Zhu, R. Guo, W. Luo, H.K. Liu, W. Jiang, S.X. Dou, J. Yang, Natl. Sci. Rev. (2020). https://doi.org/10.1093/nsr/nwaa152

[57] Z. Liu, Q. Yu, Y. Zhao, R. He, M. Xu, S. Feng, S. Li, L. Zhou, L. Mai, Chem. Soc. Rev. 48, 285 (2019)

[58] Z. Xiao, C. Lei, C. Yu, X. Chen, Z. Zhu, H. Jiang, F. Wei, Energy Storage Mater. 24, 565 (2019)

[59] J. Sun, L. Guo, X. Sun, J. Zhang, L. Hou, L. Li, S. Yang, C. Yuan, Batter. Supercaps 2, 820 (2019)

[60] R. Meng, J. Huang, Y. Feng, L. Zu, C. Peng, L. Zheng, L. Zheng, Z. Chen, G. Liu, B. Chen, Y. Mi, J. Yang, Adv. Energy Mater. 8, 1801514 (2018) 